\title{
ESTUDO LONGITUDINAL DA COMPREENSÃO VERBAL DE CRIANÇAS USUÁRIAS DE IMPLANTE COCLEAR
}

\author{
Longitudinal study of verbal comprehension of children \\ with cochlear implant
}

\author{
Carla Aparecida de Urzedo Fortunato Queiroz ${ }^{(1)}$, Maria Cecília Bevilacqua (2), \\ Maria da Piedade Resende da Costa ${ }^{(3)}$
}

\begin{abstract}
RESUMO
Objetivo: analisar a compreensão verbal de crianças surdas usuárias de implante coclear (IC) por meio de um estudo longitudinal. Métodos: os participantes foram nove crianças surdas usuárias de IC. A idade cronológica das crianças variou entre quatro e oito anos e o tempo de uso do IC foi, em média, 1 ano e 6 meses na $1^{\text {a }}$ avaliação, 3 anos e 7 meses na $2^{-}$avaliação e 4 anos e 9 meses na $3^{\text {a }}$ avaliação. As crianças foram avaliadas longitudinalmente por meio da Escala de Compreensão Verbal da RDLS. Os materiais usados foram brinquedos, objetos e figuras. Os dados foram analisados qualitativa e quantitativamente. Resultados: os resultados mostraram que as crianças implantadas obtiveram uma evolução estatisticamente significante em relação às habilidades de linguagem receptiva. Conclusão: o estudo comprova a efetividade do IC para o desenvolvimento da compreensão verbal.
\end{abstract}

DESCRITORES: Surdez; Desenvolvimento da Linguagem; Implante Coclear

\section{INTRODUÇÃO}

O Implante Coclear (IC) é atualmente o recurso tecnológico mais eficaz para favorecer o acesso da pessoa surda ao mundo sonoro. Até então nenhum dispositivo eletrônico havia possibilitado à pessoa com surdez severa/profunda adquirida antes da aquisição da linguagem, a capacidade de compreendê-la e expressá-la oralmente com funcionalidade e abstração ${ }^{1}$.

O uso do IC tem causado um dramático impacto sobre a linguagem de crianças com surdez profunda, particularmente daquelas implantadas precocemente, já que fornece o acesso a

(1) Fonoaudióloga; Pesquisadora do Centro de Pesquisas Audiológicas da Faculdade de Odontologia de Bauru da Universidade de São Paulo, CPA-FOB/USP, Bauru, SP; Doutora em Educação Especial pela Universidade Federal de São Carlos.

(2) Fonoaudióloga; Professora Titular da Faculdade de Odontologia de Bauru da Universidade de São Paulo, FOB/USP, Bauru, SP; Livre-docente.

(3) Psicóloga; Professora do Programa de Pós-Graduação em Educação Especial da Universidade Federal de São Carlos, UFSCAR, São Carlos, SP; Mestre em Educação Especial e Doutora em Psicologia Experimental.

Conflito de interesses: inexistente informações auditivas essenciais para o desenvolvimento da linguagem das crianças ${ }^{2-5}$. Ele fornece, especificamente, acesso a informações em frequências altas, o que em geral não é observado com o uso de aparelho auditivo ${ }^{6}$. Além disso, crianças implantadas apresentam melhor percepção de fala, aumento do número de vogais e consoantes produzidas e melhor inteligibilidade de fala que crianças que usam aparelho auditivo ${ }^{6,7}$. Muitos usuários de IC são capazes de se comunicar e entender a fala sem leitura orofacial e alguns conseguem falar pelo telefone. Os maiores benefícios do IC ocorrem em pessoas que adquiriram a fala e a linguagem antes da perda auditiva e/ou que possuem um tempo de privação sensorial ou tempo de surdez (anterior ao IC) curto. Todavia, um estudo longitudinal efetuado com 130 crianças com surdez pré-lingual, usuárias de IC, todas apresentaram melhoras progressivas da percepção e produção de fala, apesar do grupo de crianças implantadas precocemente ter apresentado melhor desempenho ${ }^{8}$.

É importante destacar que a habilidade de percepção de fala precede e possibilita habilidade de compreensão verbal, ou seja, a decodificação da linguagem falada. Esta, por sua vez, é imprescindível para que a expressão por meio da fala se 
desencadeie. Uma pesquisa com 181 crianças americanas e canadenses, usuárias de IC por quatro a sete anos, mostrou que mais da metade das crianças apresentou habilidades de linguagem, quanto à compreensão e expressão, similares a de seus pares ouvintes de oito a nove anos de idade. Os resultados de linguagem não foram típicos aos de crianças com perda profunda que usam apareIhos auditivos ${ }^{2}$.

Uma pesquisa com 76 crianças implantadas entre 12 e 38 meses mostrou um forte coeficiente positivo entre a linguagem falada e o tempo de uso do IC. Segundo os autores, as vantagens do uso prolongado do dispositivo para o desenvolvimento da linguagem oral são mais evidenciadas com o tempo ${ }^{4}$. Diante disso, nos grandes centros de pesquisa do mundo, os estudos longitudinais em crianças usuárias de IC têm se tornado cada vez mais importantes para comprovar e monitorar os benefícios do dispositivo para a audição e a linguagem. Um dos instrumentos usados para acompanhar o desempenho da compreensão e da expressão verbal de crianças de um a seis anos é denominado Reynell Developmental Language Scales (RDLS) ${ }^{1}$. Diversos pesquisadores têm utilizado a RDLS para avaliar o desempenho da linguagem oral de crianças usuárias de IC ao longo do tempo.

Dois grupos de crianças com surdez profunda foram avaliados por meio da RDLS. O primeiro grupo era composto por 89 crianças surdas que não usavam implantes cocleares e o segundo grupo compunha-se de 23 crianças usuárias de implantes cocleares. As crianças com implante foram avaliadas longitudinalmente: antes da implantação e após seis e 12 meses de uso do IC ${ }^{9}$. Visando verificar a influência do implante coclear para o desenvolvimento da linguagem oral, foram avaliadas 12 crianças surdas, de zero a nove anos, por meio da versão holandesa da RDLS. A RDLS foi aplicada antes da implantação, com o uso de aparelho auditivo, e após a implantação ${ }^{10}$. Um estudo longitudinal recente foi realizado com uma criança implantada, portadora de neuropatia auditiva, utilizando-se a RDLS. As autoras realizaram seis avaliações em intervalos de três meses ${ }^{11}$.

O objetivo deste trabalho foi avaliar longitudinalmente a compreensão verbal de um grupo de crianças usuárias de IC por meio da Escala de Compreensão da RDLS.

\section{MÉTODOS}

Esta pesquisa trata-se de um estudo longitudinal prospectivo realizado com um grupo de nove crianças surdas usuárias de implante coclear, dos sexos feminino e masculino. A Tabela 1 caracteriza os participantes quanto ao sexo, a idade de ativação do IC e a idade cronológica nas três avaliações. A Tabela 2 caracteriza os participantes quanto à idade auditiva, ou seja, o tempo de uso do IC.

Os materiais utilizados foram brinquedos, objetos domésticos e figuras, além de filmadora como material de apoio para a análise.

O procedimento de coleta de dados constou, primeiramente, do pedido de autorização dos pais das crianças, com assinatura de Termo de Consentimento. Posteriormente, foi aplicada a Escala de Compreensão Verbal A, da Reynell Developmental Language Scales, versão americana, adaptada para o português brasileiro ${ }^{1}$, da seguinte forma: a cada seção, algumas ordens verbais foram dadas, uma de cada vez, de forma clara e lenta, para prender a atenção da criança. Em casos de resposta errada ou parcial, a ordem não foi repetida e o resultado foi considerado negativo. Em casos de ausência de resposta, a pergunta foi repetida uma só vez. Quando a criança não respondeu adequadamente, considerou-se o item negativo. Quando solicitada a repetição da ordem pela criança, a mesma foi repetida uma única vez.

A Escala de Compreensão A é composta por 67 itens, divididos em 10 seções, sendo que cada um deles corresponde a um ponto. É importante destacar que foram avaliadas apenas as seções em que as crianças haviam falhado em pelo menos um item na avaliação anterior. Como os itens de uma determinada seção podem estar, de certa forma, correlacionados, mesmo quando a criança apresentou falha em apenas um item, toda a seção foi reavaliada. As avaliações foram realizadas nos anos 2002, 2004 e 2005.

O projeto desta pesquisa foi aprovado pelo Comitê de Ética em Pesquisa do Hospital de Reabilitação de Anomalias Craniofaciais - HRAC-USP (Ofício no. 090/2003).

O procedimento para analisar os dados constou, a princípio, de análise exploratória dos mesmos e, posteriormente, foi utilizado o modelo de regressão linear, com enfoque Bayesiano, a fim de comparar as respostas obtidas pelas crianças implantadas ao longo do tempo.

\section{RESULTADOS}

A Figura 1 mostra a resposta compreensão verbal versus tempo para as nove crianças implantadas. Nota-se, pela figura, um gráfico de configuração ascendente caracterizando o aumento gradual das pontuações de todas as crianças implantadas ao longo do tempo. 


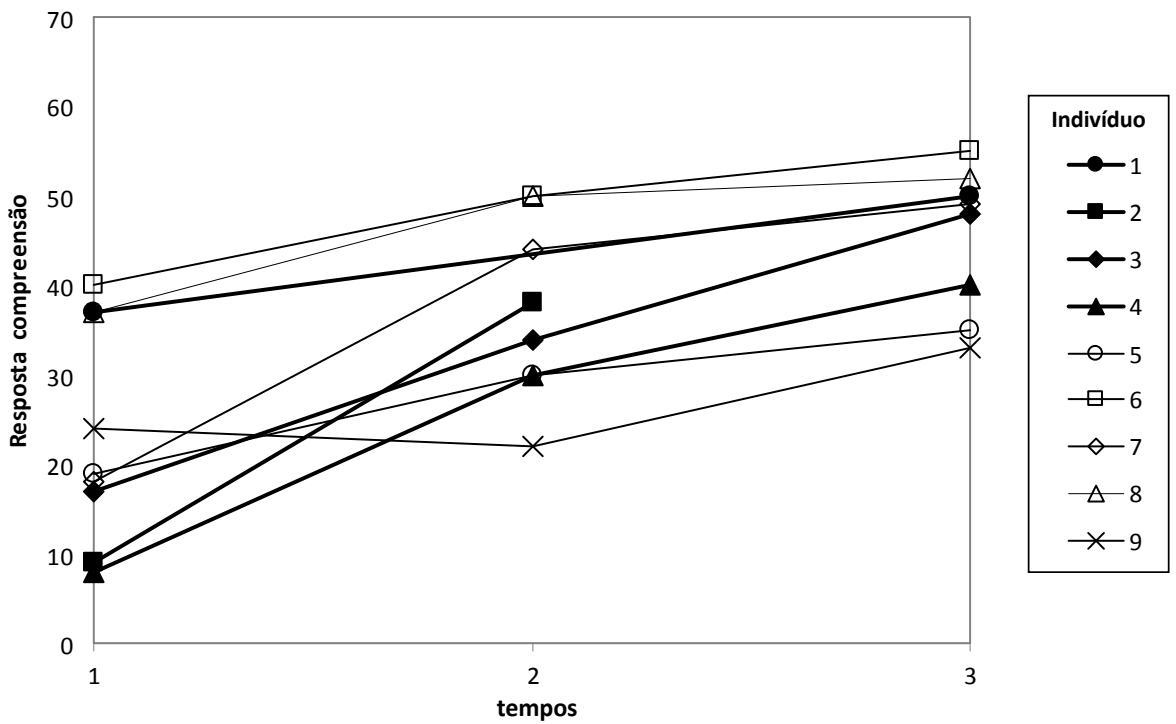

Figura 1 - Resposta compreensão verbal versus tempo para as nove crianças implantadas

Tabela 1 - Caracterização quanto ao sexo, a idade de ativação do IC e a idade cronológica das crianças

\begin{tabular}{|c|c|c|c|c|c|}
\hline \multirow{2}{*}{ Participantes } & \multirow{2}{*}{ Sexo } & \multirow{2}{*}{$\begin{array}{c}\text { Idade de } \\
\text { ativação do IC } \\
\text { (em anos) }\end{array}$} & \multicolumn{3}{|c|}{$\begin{array}{c}\text { Idade cronológica } \\
\text { (em anos) }\end{array}$} \\
\hline & & & $\begin{array}{c}\text { 19. Avaliação } \\
\text { (2002) }\end{array}$ & $\begin{array}{l}2^{\mathrm{a}} \text {. Avaliação } \\
\text { (2004) }\end{array}$ & $\begin{array}{l}\text { 3á. Avaliação } \\
\text { (2005) }\end{array}$ \\
\hline 1 & $\mathrm{~F}$ & $2: 10$ & $4: 3$ & $\ldots$ & $7: 2$ \\
\hline 2 & $M$ & $3: 2$ & $4: 5$ & $6: 4$ & $\ldots$ \\
\hline 3 & $M$ & $3: 5$ & $4: 6$ & $6: 6$ & $7: 11$ \\
\hline 4 & $F$ & $4: 0$ & $4: 7$ & $6: 6$ & $7: 6$ \\
\hline 5 & $M$ & $2: 9$ & $4: 6$ & $6: 9$ & $7: 8$ \\
\hline 6 & $F$ & $2: 0$ & $4: 8$ & $6: 10$ & $7: 10$ \\
\hline 7 & $M$ & $3: 6$ & $4: 9$ & $6: 10$ & 8:0 \\
\hline 8 & $\mathrm{~F}$ & $2: 8$ & $4: 10$ & $7: 0$ & $8: 2$ \\
\hline 9 & $M$ & $3: 7$ & 5:0 & $7: 2$ & $8: 3$ \\
\hline
\end{tabular}

Tabela 2 - Caracterização dos participantes quanto à idade auditiva

\begin{tabular}{cccc}
\hline & \multicolumn{3}{c}{$\begin{array}{c}\text { Idade auditiva ou tempo de uso do IC } \\
\text { Em anos (em meses) }\end{array}$} \\
\cline { 2 - 4 } Participantes & $\begin{array}{c}1^{\text {a }} \text {. Avaliação } \\
2002\end{array}$ & $\begin{array}{c}2^{\text {a }} \text {. Avaliação } \\
2004\end{array}$ & $\begin{array}{c}3^{\text {a }} \text {. Avaliação } \\
2005\end{array}$ \\
\hline 1 & $1: 5(17 \mathrm{~m})$ & $\ldots$ & $4: 4(52 \mathrm{~m})$ \\
2 & $1: 3(15 \mathrm{~m})$ & $3: 2(38 \mathrm{~m})$ & $\ldots$ \\
3 & $1: 1(13 \mathrm{~m})$ & $3: 1(37 \mathrm{~m})$ & $4: 6(54 \mathrm{~m})$ \\
4 & $0: 7(7 \mathrm{~m})$ & $2: 6(28 \mathrm{~m})$ & $3: 6(42 \mathrm{~m})$ \\
5 & $1: 7(19 \mathrm{~m})$ & $4: 0(48 \mathrm{~m})$ & $4: 11(59 \mathrm{~m})$ \\
6 & $2: 8(32 \mathrm{~m})$ & $4: 10(58 \mathrm{~m})$ & $5: 10(70 \mathrm{~m})$ \\
7 & $1: 3(15 \mathrm{~m})$ & $3: 4(40 \mathrm{~m})$ & $4: 6(54 \mathrm{~m})$ \\
8 & $2: 2(26 \mathrm{~m})$ & $4: 4(52 \mathrm{~m})$ & $5: 6(66 \mathrm{~m})$ \\
9 & $1: 5(17 \mathrm{~m})$ & $3: 7(43 \mathrm{~m})$ & $4: 9(57 \mathrm{~m})$ \\
Médias & $1: 6(18 \mathrm{~m})$ & $3: 7(43 \mathrm{~m})$ & $4: 9(57 \mathrm{~m})$ \\
\hline
\end{tabular}


A Tabela 3 mostra a média, o desvio padrão e a mediana para a variável compreensão verbal do grupo das crianças surdas usuárias de IC nos Tempos 1,2 e 3 . Verifica-se, pela tabela, o aumento das médias e medianas das pontuações das crianças implantadas no decorrer dos tempos demonstrando melhora nas habilidades de compreensão verbal.

A Tabela 4 mostra as comparações entre as diferenças entre os "tempos", ou seja, os resultados das três avaliações para a variável compreensão e os seus respectivos intervalos de credibilidade. Considera-se diferença significante quando o zero não está contido nos intervalos de credibilidade. Nota-se, portanto, que houve diferença significante ao se comparar os tempos, ou seja, as avaliações realizadas longitudinalmente para a variável compreensão. $\mathrm{O}$ aumento das pontuações das crianças ao longo dos tempos, ou seja, a evolução da compreensão verbal graças ao uso do IC foi considerável e comprovada estatisticamente.

Tabela 3 - Média, desvio padrão (D.P.) e mediana para a variável compreensão das crianças implantadas segundo os tempos

\begin{tabular}{ccccc}
\hline & & \multicolumn{3}{c}{ Compreensão } \\
\hline Tempos & $\mathbf{n}$ & Média & D.P. & Mediana \\
\hline Tempo 1 & 9 & 23,0 & 11,86 & 19,00 \\
Tempo 2 & 8 & 40,38 & 9,21 & 39,00 \\
Tempo 3 & 8 & 51,63 & 8,14 & 55,50 \\
\hline
\end{tabular}

Tabela 4 - Comparações dos tempos para a variável compreensão e respectivos intervalos de credibilidade $\left(\mathrm{IC}_{\mathrm{r}}\right)$

\begin{tabular}{ccc}
\hline Comparação & Estimativas & $\mathbf{I C}_{\mathbf{r}}(\mathbf{9 5 \%})$ \\
\hline Tempo1 - Tempo2 & $-18,38$ & $(-24,32 ;-12,42)^{(\mathrm{a})}$ \\
Tempo1 - Tempo3 & $-27,85$ & $(-33,89 ;-21,93)^{(\mathrm{a})}$ \\
Tempo2 - Tempo3 & $-9,48$ & $(-15,72 ;-3,25)^{(\mathrm{a})}$ \\
\hline
\end{tabular}

(a) Intervalo que o zero não está contido

\section{DISCUSSÃO}

Os resultados apresentados mostraram a evolução das crianças surdas implantadas, participantes deste estudo, quanto à compreensão verbal ao longo do tempo. Isso é claramente evidenciado por meio da Figura 1, que mostra as respostas para compreensão verbal versus os tempos em que as avaliações foram realizadas. Resultados similares foram obtidos em estudo com uma criança implantada avaliada por meio da RDLS ${ }^{11}$. As autoras do referido estudo obtiveram curva ascendente de evolução de linguagem tanto para a escala de expressão como para a de compreensão, significando avanço no desenvolvimento da linguagem.

O aumento das médias e medianas das pontuações das crianças implantadas no decorrer dos tempos e a diferença entre os mesmos demonstraram que houve uma evolução estatisticamente significante em relação à compreensão verbal, ou seja, as diferenças entre as pontuações demonstraram valores consideráveis. Pode-se, portanto, afirmar que houve uma melhora de desempenho nítido e comprovado quanto à linguagem receptiva deste grupo de crianças usuárias de IC.

Em estudo com a versão holandesa da RDLS, crianças surdas foram avaliadas antes da implantação, com o uso de aparelho auditivo, e após a implantação. Os resultados foram comparados, observando um aumento das pontuações quanto à compreensão e expressão verbal ${ }^{10}$.

Em estudo efetuado com 106 crianças com pelo menos dois anos de uso do IC, o desenvolvimento da linguagem receptiva e expressiva foi avaliado por meio das Escalas de habilidades comunicativas para crianças surdas e por meio da RDLS III. Os resultados pré e pós-implantação foram comparados estatisticamente, e uma análise de regressão linear foi efetuada. Os resultados mostraram melhora considerável da percepção, da compreensão e produção de fala ${ }^{12}$. 
Outro estudo longitudinal com 23 crianças surdas usuárias de IC, avaliadas com a Escala Reynell antes da implantação (com AASIs) e seis meses após a implantação, observaram melhoras significativas ao longo do tempo para a compreensão da linguagem ${ }^{13}$.

Crianças surdas com IC e sem IC foram avaliadas longitudinalmente. Os resultados obtidos pelas crianças sem implante por meio das Escalas Reynell mostraram uma regressão por idade. Elas obtiveram metade ou menos da metade dos resultados obtidos pelos seus pares ouvintes. A média do desenvolvimento da linguagem para a compreensão das crianças implantadas no primeiro ano de uso do IC foi equivalente ao das crianças ouvintes ${ }^{9}$, demonstrando a evolução da compreensão e expressão verbal ao longo do tempo.

Conforme já mencionado, é frequentemente destacado na literatura um coeficiente positivo entre o tempo de uso do IC e a linguagem oral das crianças, indicando que quanto maior o tempo de uso, melhor a linguagem. A evolução das habilidades de linguagem pode ser observada para cada mês adicional de uso do IC após os 12 primeiros meses de uso ${ }^{4,14}$.

Os resultados apresentados neste estudo, em concordância com a literatura nacional e internacional, enfatizaram a efetividade do implante coclear para o acesso da criança surda aos sons de fala e conseqüente habilidade de compreendê-los ao longo do tempo. O estudo mostrou a efetividade do IC não apenas para perceber, detectar e reconhecer a fala, como já comprovado pela literatura ${ }^{8,14,15}$, mas também para uma habilidade auditiva mais complexa e refinada, ou seja, a capacidade de compreender verbalmente a linguagem. Essa "capacidade" iniciou-se com o reconhecimento simples de palavras e evoluiu para o entendimento de ordens verbais mais complexas e abstratas.

As necessidades de ordem clínica têm motivado pesquisadores ao aperfeiçoamento de procedimentos específicos para a avaliação da linguagem de crianças implantadas ${ }^{16}$, uma vez que há muitos trabalhos sobre a audição nas crianças com implante, mas poucos sobre a sua linguagem ${ }^{17}$, considerando-a como uma atividade constitutiva, interacional e subjetiva.

A avaliação e follow-up das habilidades de linguagem e comunicação em crianças implantadas são importantes por diversos motivos, dentre eles, a verificação da efetividade do IC e do progresso do desempenho auditivo ${ }^{18}$. É relevante enfatizar que os estudos continuados da linguagem além de evidenciar, documentar e caracterizar os benefícios propiciados pelo uso sistemático do IC, favorecem a intervenção fonoaudiológica e educacional, tendo como foco um adequado e favorável desenvolvimento da linguagem.

\section{CONCLUSÃO}

O estudo longitudinal em questão demonstrou que o uso sistematizado do implante coclear propicia a evolução da habilidade de compreensão verbal em crianças usuárias do dispositivo, sendo esta habilidade imprescindível para que a criança se expresse por meio da fala.

\section{AGRADECIMENTOS}

À CAPES, pelo apoio financeiro concedido.

\begin{abstract}
Purpose: to analyze the verbal comprehension of deaf children using a cochlear implant $(\mathrm{Cl})$ through a longitudinal study. Methods: the individuals were nine deaf children using $\mathrm{Cl}$. The chronological age of the children ranged from four to eight year-old. The time of use of $\mathrm{Cl}$ was, on average, 1 year 6 months at the $1^{\text {st }}$ evaluation, 3 years 7 months at the $2^{\text {nd }}$ evaluation, and 4 years 9 months at the $3^{\text {rd }}$ evaluation. The deaf children were evaluated longitudinally through the RDLS Verbal Comprehension Scale. The used materials were toys, objects and figures. Data were analyzed qualitatively and quantitatively. Results: the implanted children showed progress in their receptive abilities that were statistically significant. Conclusion: the study proves $\mathrm{Cl}$ effectiveness as for the development of verbal comprehension.
\end{abstract}

KEYWORDS: Deafness; Language Development; Cochlear Implantation 


\section{REFERÊNCIAS}

1. Fortunato-Queiroz CAU. Reynell Developmental Language Scales (RDLS): um estudo longitudinal em crianças usuárias de implante coclear. [tese] São Carlos (SP): Universidade Federal de São Carlos; 2007.

2. Geers AE, Nicholas JG, Sedey AL. Language skills of children with early cochlear implantation. Ear Hear. 2003; 24(Suppl1):46S-58S.

3. Harrison RV, Gordon KA, Mount RJ. Is there a critical period for cochlear implantation in congenitally deaf children? Analyses of hearing and speech perception performance after implantation. Develop Psychobiol. 2005; 46(3):252-61.

4. Nicholas JG, Geers AE. Effects of early auditory experience on the spoken language of deaf children at 3 years of age. Ear Hear. 2006; 27(3):286-98.

5. Nicholas JG, Geers AE. Will they catch up? The role of age at cochlear implantation in the spoken language development of children with severe to profound hearing loss. J Speech Lang Hear Res. 2007; 50(4):1048-62.

6. Bollard PM, Chute PM, Popp A, Parisier SC. Specific language growth in young children using the CLARION cochlear implant. Ann Otol Rhinol Laryngol Suppl. 1999; 177:119-23.

7. Hay-McCutcheon MJ, Kirk KI, Henning SC, Gao S, Qi R. Using early language outcomes to predict later language ability in children with cochlear implants. Audiol Neurootol. 2008; 13(6):370-8.

8. Manrique M, Cervera-Paz FJ, Huarte A, Martínez I, Gomez A, Vázquez de la Iglesia F. [Hearing and speech in children under 2 years of age with a cochlear implant]. An Sist Sanit Navar. 2004; 27(3):305-17.

9. Robbins AM, Svirsky M, Kirk KI. Children with implants can speak, but can they communicate? Otolaryngol Head Neck Surg. 1997; 117(3Pt1):155-60.
10. Miyamoto RT, Kirk KI, Svirsky MA, Sehgal ST. Communication skills in pediatric cochlear implant recipients. Acta Otolaryngol (Stockh). 1999; 119(2):219-24.

11. Resegue MM, Moret ALM, Bevilacqua MC. Neuropatia auditiva: aspectos do desenvolvimento de linguagem em uma criança usuária de implante coclear. In: Anais do $22^{\circ}$ Encontro Internacional de Audiologia; 2007 março 28-31; Natal, Brasil. 22을 Encontro Internacional de Audiologia; 2007.

12. Richter B, Eissele S, Laszig R, Löhle E. Receptive and expressive language skills of 106 children with a minimum of 2 years' experience in hearing with a cochlear implant. Int J Pediatr Otorhinolaryngol. 2002; 64(2):111-25.

13. Robbins AM, Bollard PM, Green J. Language development in children implanted with the CLARION cochlear implant. Ann Otol Rhinol Laryngol Suppl. 1999; 177:113-8.

14. Moret ALM, Bevilacqua MC, Costa OA. Implante coclear: audição e linguagem em crianças deficientes auditivas pré-linguais. Pró-Fono. 2007; 19(3):295-304.

15. Colletti V, Carner M, Miorelli V, Guida M, Colletti L, Fiorino FG. Cochlear implantation at under 12 months: report on 10 patients. Laryngoscope. 2005; 115(3):445-9.

16. Padovani CMA, Teixeira ER. Using the MacArthur Comunicative Development Inventories (CDI'S) to assess the lexical development of cochlear implanted children. Pró-Fono. 2004; 16(2):217-24.

17. Santana AP. O processo de aquisição da linguagem: estudo comparativo de duas crianças usuárias de implante coclear. Dist Comun. 2005; 17(2):233-43.

18. Mylanus E, Vermeulen A, Neijenhuis K, Snik A. Auditory and language assessment tools in pediatric cochlear implant patients: a follow-up study. In: Abstracts of the $8^{\text {a }}$ European Symposium Pediatric Cochlear Implants; 2006 Mar 25-28; Palazzo del Cinema. Lido di Venezia; 2006. p.106.

RECEBIDO EM: 02/12/2008

ACEITO EM: 31/08/2009

Endereço para correspondência:

Carla Aparecida de Urzedo Fortunato

Rua Zina Olga Caldo Donato, 525

Ribeirão Preto - SP

CEP: $14065-260$

E-mail: caurzedo@yahoo.com 